\title{
HIGH-DENSITY ARRAYS OF VALVES AND INTERCONNECTS FOR LIQUID SWITCHING
}

\author{
Luc Bousse*, Emiel Dijkstra**, and Olivier Guenat*** \\ Molecular Devices Corp. \\ 1311 Orleans Drive, Sunnyvale, CA 94025
}

\begin{abstract}
We describe a process for the fabrication of high-density arrays of valves designed for switching liquids. To make small valves capable of opening and closing large orifices for liquid flow requires a flexible movable membrane. We use silicone rubber, because it is elastomeric, makes good seals, and can be spin-coated. Flow channels are defined by a sacrificial photoresist. The behavior of individual valves is measured, and a model describing the flow is presented. Valve arrays at densities up to 16 valves $/ \mathrm{cm}^{2}$ have been demonstrated.

\section{INTRODUCTION}

Previously, we have described an eight-channel chip with 32 sensing spots for the measurement of the metabolic responses of living cells [1]. To improve time response and reduce the sample volume, we need an array of eight four-way valves on a chip that can be directly interfaced with the sensing chip. The type of interface that minimizes dead volume is to stack the valve chip and the sensor chip directly on top of each other. This defines the valve density required; since a fourway valve can be implemented with four one-way valves, we need 32 valves in the area of the chip, which is $5.3 \mathrm{~cm}^{2}$. This defines the required valve density as at least 6 valves $/ \mathrm{cm}^{2}$; however, the constraint is even greater since the placement is not arbirary. A density of 10 to $20 \mathrm{valves} / \mathrm{cm}^{2}$ is desirable, and a smallest dimension of less than $1 \mathrm{~mm}$.

Most microfabricated valves described previously work only with gases and are 5-8 $\mathrm{mm}$ on a side for a single valve, meaning maximum densities of 4 valves $/ \mathrm{cm}^{2}$. The aim of this work is to develop a technology for making high density arrays of valves for liquids. This must include an interconnection method for the individual components. Although the design specifications follow from a specific application, the process and devices presented here are not limited to this applicaion, but can be used for a wide variety of purposes.
\end{abstract}

\section{CHOICE OF MEMBRANE MATERIAL}

To make valves smaller than $1 \mathrm{~mm}$, yet maintain openings of about $100 \mu \mathrm{m}$ for liquid flow requires a movable membrane material much more flexible than glass or silicon and capable of large deformations. For instance, to deflect a membrane $500 \mu \mathrm{m}$ wide by $100 \mu \mathrm{m}$ in the middle, an elongation of about $15 \%$ is needed (assuming the deflected shape is an ellipse). To avoid large forces in causing these displacements, a low Young's modulus is needed. One material with a low Young's modulus is polyimide $\left(E\right.$ is $3 \times 10^{3} \mathrm{MPa}$, as opposed to $1.9 \times 10^{5} \mathrm{MPa}$ for silicon), which has been used for micropump membranes [2]. However, polyimide is not capable of large elongations. A class of materials with even lower modulus values are the elastomers, such as silicone rubber, which have also been used for microvalve membranes [3]. We chose silicone rubber because it has a very low modulus, is an elastomer which forms very good seals, and can be spin coated and cured on silicon wafers. Most types of mechanical actua- tors can be adapted to our valves, but for our application the most convenient and flexible is pneumatic acruation.

\section{PROCESS DESCRIPTION}

Given that we desire an elastomeric membrane, it is desirable to make these membranes a permanent part of the valve structure. Handling loose membranes to place in some assembly is difficult, and cannot be done on a wafer scale. Therefore, we spin-coat the uncured polymer on the silicon wafer, and then cure it at $150^{\circ} \mathrm{C}$. By using an addition-cure two-component rubber, this forms a very tight bond between the rubber and the oxidized silicon. To form flow channels between the rubber and the silicon, a sacrificial layer is needed to avoid the bonding of rubber to the substrate in some areas. The simplest type of sacrificial layer is photolithographically pattemed photoresist. Subsequent removal of the sacrificial layer will then create the regions where flow can occur. To provide access to the flow channel, holes have to be made through the wafer. To do this, we used anisoropic etching, which can terminate gently on the oxide layer on the other of the wafer. This overall process is similar to that described by Shoji [4] for devices in which the membrane is negative photoresist. The detailed description of the process steps is :

1. Staring material: $100 \mathrm{~mm}$ diameter low-doped silicon wafers, double-sided polished, $500 \mu \mathrm{m}$ thick.

2. Wet oxidation to form a $1 \mu \mathrm{m}$ thick oxide on both sides.
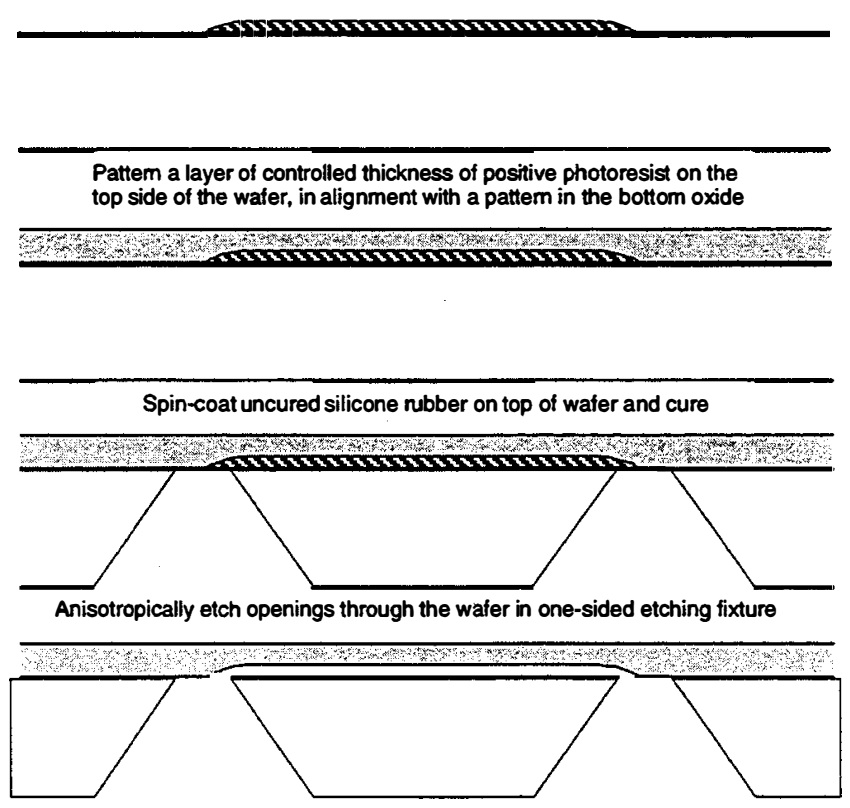

Etch oxide and remove sacrificial photoresist

Figure 1. Process schematic 


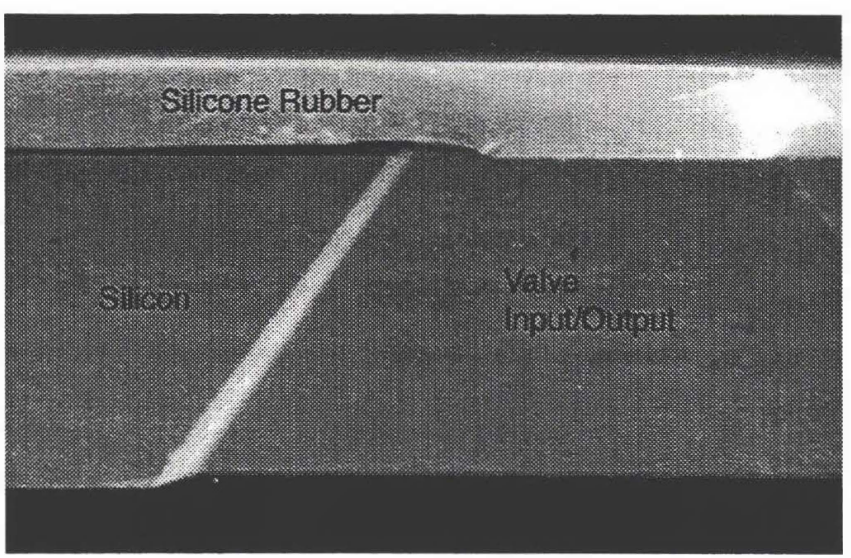

Figure 2. SEM close-up of the cross-section of a valve structure.

3. Pattern backside oxide with the interconnection channel pattern.

4. Anisotropically etch interconnection channel to desired depth.

5. Strip oxide and regrow it.

6. Photolithographically define openings in the oxide on the backside, for the holes through the wafer.

7. Spin-coat sacrificial photoresist on the frontside of the wafer, in alignment with the hole pattern on the backside.

8. Spin-coat uncured silicone rubber (Dow-Coming 96-083) on the frontside, on top of the photoresist, and cure (1 hour at $150^{\circ} \mathrm{C}$ ).

9. Etch holes in the silicon from the bottom in an anisorropic etchant. This is done in a one-sided silicon etch fixture, so that the rubber on the frontside of the wafer is not damaged by the etchant. This etch step self-terminates when the oxide on the other side of the wafer is reached.

10. Etch the oxide windows in buffered $\mathrm{HF}$, which also removes all the oxide exposed on the backside.

11. Remove the sacrificial photoresist layer in a resist removing solution.

12. Wafer dicing.

Fig. 1 shows the key steps in this process, but without including the interconnection channel. Fig. 2 shows an SEM close-up of the crosssection of an actual structure. The top geometry is defined in Fig. 3.

\section{A SIMPLE MODEL TO \\ DESCRIBE VALVE OPERATION}

In this section, we will develop an analytical model for the flow $Q$ in a valve structure such as shown in Fig. 1, as a function of the applied pressures. Those pressures are the driving pressure on the inlet side $P_{d r}$, and the pneumatic actuation pressure applied to close the valve $P_{c l}$. The pressure at the outlet is assumed to be zero, without loss of generality. The dimensions of the flow channel are typically $2000 \mu \mathrm{m}$ in length, 500 to $1500 \mu \mathrm{m}$ in width, and 0 to $100 \mu \mathrm{m}$ in height, depending on the applied pressure. It is natural to model the flow in such a long and thin channel as the laminar flow between two parallel plates, which is given by:

$$
Q=\frac{1}{12 \mu} \frac{W H^{3}}{L} P_{d r}
$$

where the dimensions $\mathrm{W}$ and $\mathrm{L}$ are defined in Fig. $3, \mu$ is the dynamic viscosity of the fluid, and $H$ is the height of the flow channel. This height depends on the displacement of the silicone rubber membrane due to the net pressure acting on it, namely $\Delta P=P_{d r}-P_{c l}$. The displacement of a rectangular membrane of width $W$ and length $L$ is given by [5]:

$$
\Delta P=\frac{C_{1} \sigma}{a^{2}} d+\frac{C_{2} E t}{a^{4}} d^{3}
$$

where $2 a$ is the smaller of $W$ and $L, E$ is Young's modulus, $\sigma$ the residual stress, $t$ the thickness of the membrane, and $C_{1}$ and $C_{2}$ are constants dependent on the ratio $W / L$ and Poisson's ratio $v$. In the case of an elastomeric membrane, Young's modulus is very low, and thus the linear term is dominant (see measurements below). We will therefore assume that the membrane moves linearly with applied pressure, and we will call the proportionality constant $\alpha$.

$$
H=\alpha\left(P_{d r}-P_{c l}\right)
$$

This equation neglects the contribution to $H$ due to the sacrificial layer itself, which is usually much smaller than the displacement caused by pressure. Substituting this equation into (1) we obtain:

$$
Q=\frac{1}{12 \mu} \frac{W}{L} \alpha^{3}\left(P_{d r}-P_{c l}\right)^{3} P_{d r}
$$

When $P_{d r} \gg P_{c l}$ this can be approximated as:

$$
Q=\frac{1}{12 \mu} \frac{W}{L} \alpha^{3}\left(P_{d r}-P_{c l}\right)^{4}
$$

although this approximation turns out to be useful in a wider range of pressures as well. Both equations (4) and (5) predict that flow will increase very rapidly with driving pressure, due to the flexibility of the membrane. When $Q$ is high, however, the pressure loss in the openings through the wafer, and in the fittings used to access the valve is no longer negligible. This must be modeled by a flow resistance in series with the valve itself. This situation is similar to modeling the currentvoltage characteristics of a semiconductor $\mathrm{p}-\mathrm{n}$ junction: at high currents the effect of the series resistance of the semiconductor becomes dominant. If we call the externally measured pressure $P_{\text {ext }}$, then we can write:

$$
P_{\text {ext }}=R Q+P_{d r}
$$

where $R$ is the flow resistance in series with the valve. The value of the approximation of equation (5) is that it is now possible to invert that equation to obtain:

$$
P_{e x t}=R Q+P_{c l}+\left(\frac{12 \mu}{(W / L) \alpha^{3}}\right)^{1 / 4} Q^{1 / 4}
$$

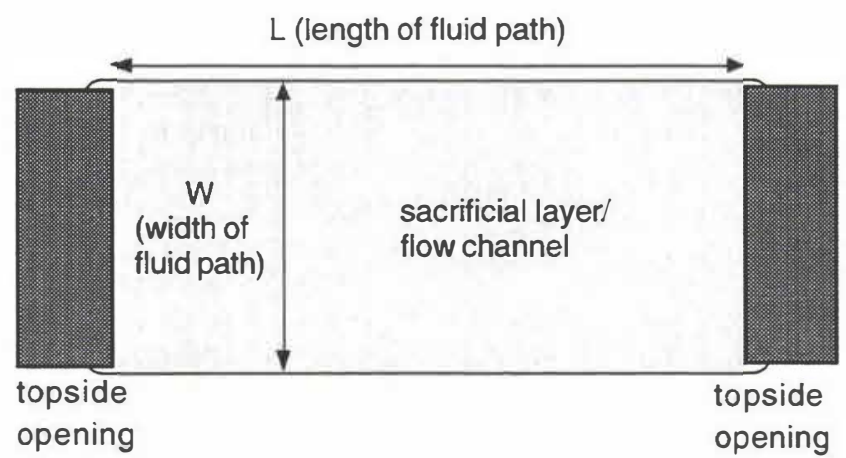

Figure 3. Definition of top geometry. 


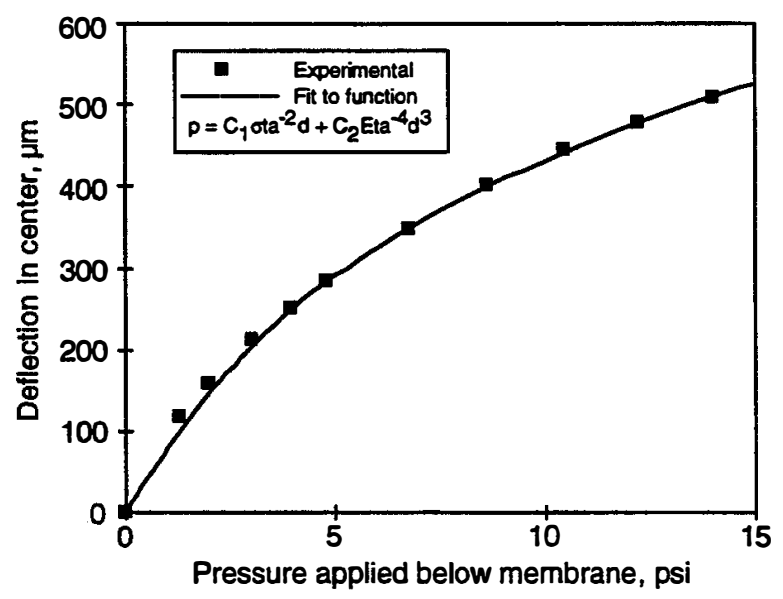

Figure 4. Experimental and theoretical load-deflection curves.

This equation models the entire family of curves of $Q$ as a function of $P_{e r s}$ and $P_{d r}$, with two adjustable parameters, namely $\alpha$ and $R$.

\section{MEASUREMENTS ON INDIVIDUAL VALVES}

The first type of measurements we will present here are the loaddeflection curves of the membranes as a pressure is applied underneath. These measurements, although they do not directly test the valve function, are useful on two levels. First, they allow the characterization of the silicone nubber membranes, in particular the residual tensile stress that keeps them stretched out. Second, by directly measuring the slope $\alpha$ used in Eq. (3), it is an important verification of the model presented in the previous section. Although we made some special-purpose square test structures similar to those used by Senturia et al. [6] for polyimide characterization, it turned out that a valve structure can be used as well, since pressure can be applied to the entire membrane through the two holes through the wafer. In that way, the same device can be used for measuring $\alpha$ with two different methods.

The device was tested in a fixture that allowed free observation of the top of the membrane, and the application of pressure at the bottom. Pressures were measured with solid-state pressure ransducers, and the deflection height of the center of the membrane was measured with the calibrated focus of a microscope. The thickness of the silicone rubber was measured by looking at the diced cross-section at the edge of the chip with a calibrated microscope. Fig. 4 shows the data on a device with $W=1239 \mu \mathrm{m}, L=2428 \mu \mathrm{m}$, and membrane thickness $58 \mu \mathrm{m}$. A good fit with the function of Eq. (2) is obtained, and the linear term corresponds to $\alpha_{\mathrm{ld}}=81.8 \mu \mathrm{m} / \mathrm{psi}$. The linear and cubic coefficients can be used together with Eq. (2) to give Young's modulus $E=0.87 \mathrm{MPa}$ and the residual stress $\sigma=0.28 \mathrm{MPa}$. This corresponds to a quite large residual stress of $32 \%$. We encountered values of $E$ from 0.7 to 1.0 $\mathrm{MPa}$, and $\sigma$ from 0.23 to $0.28 \mathrm{MPa}$, while testing a range of samples with thicknesses from 29 to $107 \mu \mathrm{m}$. The thickness did not appear to affect either $E$ or $\sigma$ significantly.

The next type of test is to measure how well these structures work as valves. A different test fixture was used for this, one that included an external gasket to separate the two flow openings. Driving pressure was applied by pressurizing a vessel filled with deionized water. All tests were done at room temperature with filtered DI water. Flow was measured gravimetrically for high flow rates, and by bubble movement in a calibrated capillary for low flow rates. Fig. 5 shows data obtained on the same structure as used for Fig. 4, namely flow as a function of driving pressure, with closing pressure as a parameter. Each curve has an initial domain where $Q$ increases very rapidly with pressure, and then the dependence becomes linear as the external flow resistances become dominant. The value of $\alpha$ found by fitting these curves to Eq. (7) is $65.3 \mu \mathrm{m} / \mathrm{psi}$. As expected, this is somewhat smaller than $\alpha_{l d}$, since the channel height in Eq. (1) is an average, not the maximum found in the middle of the membrane. Taking account of this, the two values of $\alpha$ agree very well.

Eq. (7) predicts that applying a closing pressure shifts the curves by the same amount as the closing pressure. The dotted curves in Fig. (5) show how well that model works when higher closing pressures are applied. As expected, when $P_{c l}$ is 2 psi or greater, the agreement with data becomes poor. There are two possible reasons for this: first, the approximation leading to Eq. (5) is not valid anymore. Second, Eq.(3) is not entirely correct, since the closing pressure is applied uniformly to the top of the membrane, while the driving pressure is only applied to one edge of the membrane. A simple way of modeling this is to assume that the average value of the pressure under the membrane in the channel is not $P_{d r}$ but rather $P_{d r} / \beta$. This leads to Eq. (7) being modified to:

$$
P_{e x t}=R Q+\beta P_{c l}+\beta\left(\frac{12 \mu}{(W / L) \alpha^{3}}\right)^{1 / 4} Q^{1 / 4}
$$

The continuous line in Fig. (5) represents this model with three adjustable parameters, where $\beta=1.2$. Clearly, adding this parameter improves the fit of the model considerably.

Another way of looking at valve characteristics is as a function of closing pressure, for a fixed driving pressure. This shows how well a valve can shut off flow, and is best seen in logarithmic coordinates. Fig. (6) shows data in this way for three different valves that have the same $W$ and $L$, but different thicknesses of the sacrificial layer. Clearly, thinner sacrificial layers produce much lower leakage flows. This is probably due to leakage at the edge of the flow channel, which does not entirely collapse when closing pressure is applied. This can be avoided by extending the sacrificial layer beyond the top openings of the flow holes.

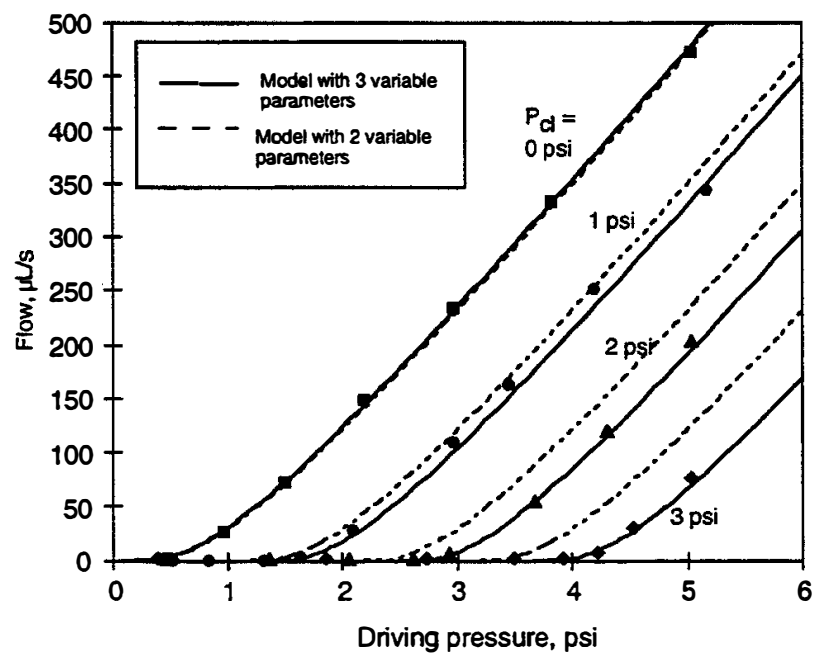

Figure 5. Flow as a function of driving pressure with closing pressure as a parameter. 


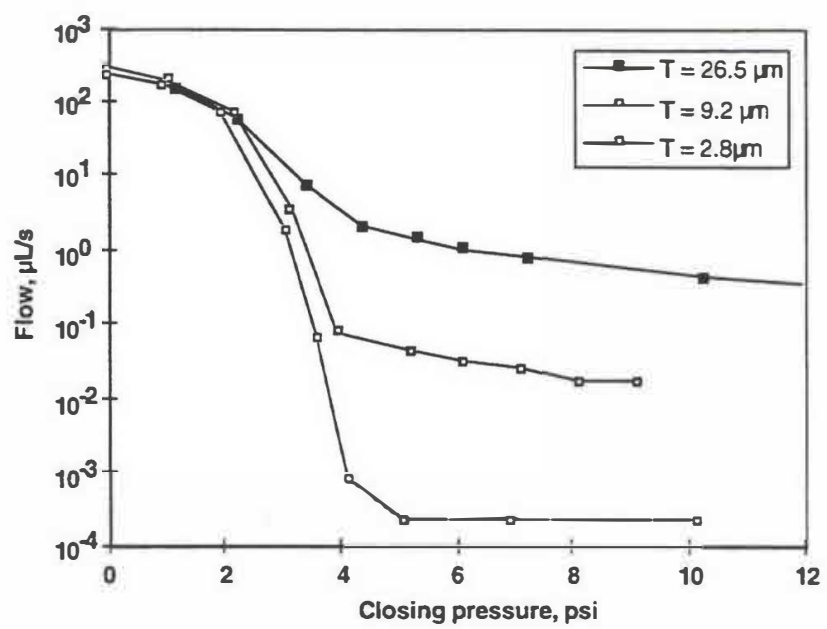

Figure 6. Flow as a function of closing pressure for a fixed 4 psi driving pressure for three sacrificial layer thicknesses.

\section{TESTING OF INTEGRATED VALVE ARRAYS}

The true advantages of this integrated valve technology are only realized when use is made of the fluid interconnect layer at the bottom of the wafer. This allows valves to be interconnected in any pattern that is allowable in two dimensions. In particular, for the applications we were pursuing, the most useful structure is a four way valve, made with four individual valves, but with inputs and outputs combined, as shown in Fig. (7). In addition to testing the individual valves that make up this array, we have tested the ability of the array to switch flow from one path to another. The setup of that experiment and its results are shown in Fig. 8. Two of the valves are constantly held closed, while 2 psi driving pressure is applied to the input located between the two other valves. As the closing pressure is gradually switched from one valve to the other, the flow also is switched. Note that in Fig. 8 the flow on/flow off ratio is 106 ; this is due in part to the improved layout seen in Fig. 7, where the sacrificial layer extends beyond the top openings. The density of the structure of Fig. 7 is about 16 valves $/ \mathrm{cm}^{2}$; the densest structures we have fabricated are a set of three four way valves in a $1 \mathrm{~cm}^{2}$ chip, for a density of 12 valves $/ \mathrm{cm}^{2}$.

\section{DISCUSSION AND CONCLUSION}

Comparing this work to the recently reported work by Vieider $e t$ al., [3] who also used silicone rubber membranes, there are several important differences. In this paper, the flow path and the movable membrane are integrated in a single die; in Ref. [3] two chips must be aligned in an external fixture. The flow path is much shorter in [3], leading to a valve with a rapid transition between open and closed states; the valves described here have a longer flow path, which lends itself more to accurate flow control.

The integration of the movable membrane in the flow chip is achieved with a sacrificial layer; one of the problems we encountered after removing that layer is, not surprisingly, stiction. In most cases, acetone or isopropanol were needed to initially unstick membranes. More work will be needed to devise methods to overcome this problem. There are many further directions into which this work can be taken. One of them is to combine these structures with other actuation methods. Another is to integrate the flow inputoutput manifolds, and the closing pressure manifold, so that valve chips need no further external fixtures to operate.

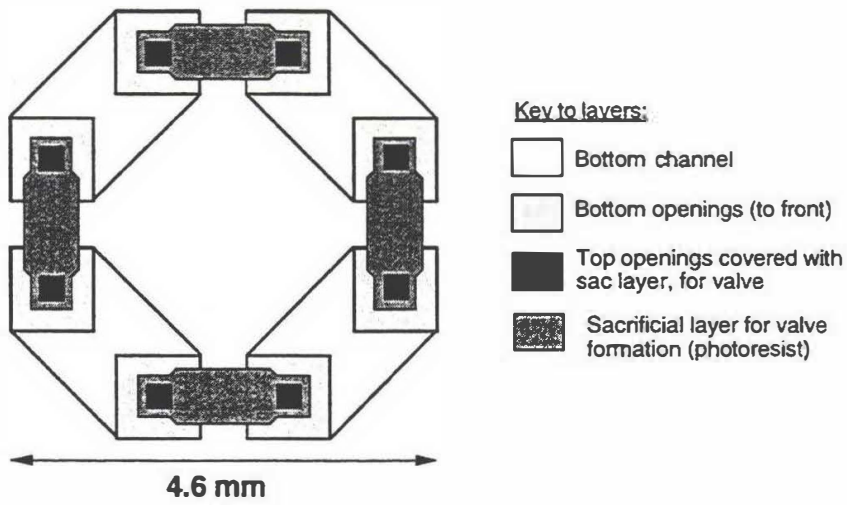

Figure 7. Layout of a symmetrical four-way valve array, made with four one-way valves. The size is $4.6 \mathrm{~mm}$ on a side. Other, non-symmetrical, layouts that minimize the size along one dimension hove also been fabricated.

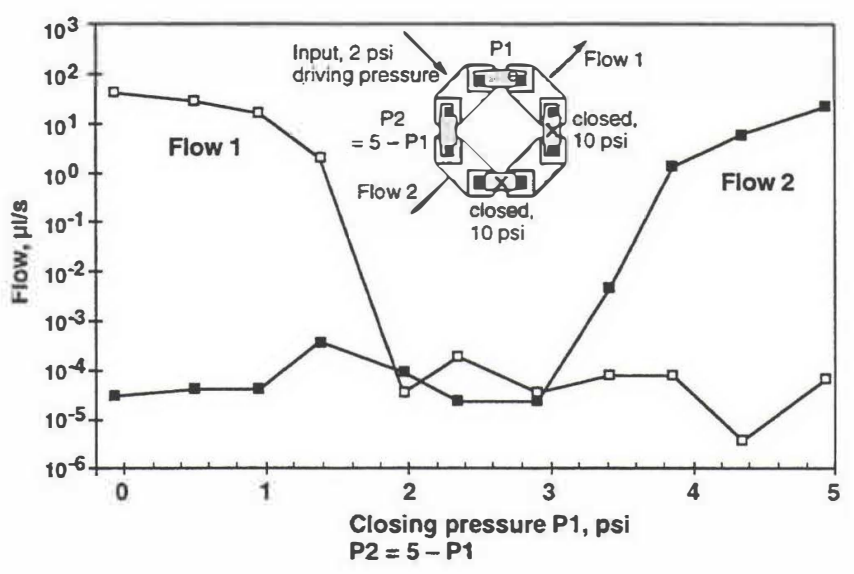

Figure 8. Test results of the valve array shown in Figure 7

\section{REFERENCES}

1. L. Bousse, "Whole cell biosensors", Proceedings of Transducers 95, Vol. 2, Stockholm (1995) p. 483.

2. W.K. Schomburg, B. Büstgens, J Fahrenberg, and D. Maas, "Components for microfluidic handling modules", Proceedings of the $\mu T A S$ '94 Workshop, Kluwer (1995) p. 255.

3. C. Vieider, O. Öhman, and H. Elderstig, "A pneumatically actuated micro valve with a silicone rubber membrane for integration with fluid handling systems", Proceedings of Transducers 95, Vol. 2, Stockholm (1995), p. 284.

4. S. Shoiji, B. Van der Schoot, N. de Rooij, and M. Esashi, "Smallest dead volume microvalves for integrated chemical analyzing systems", Proceedings of Transducers 91, San Francisco (1991), p. 1052.

5. O. Tabata, K. Kawahata, S. Sugiyama, and I. Igarashi, "Mechanical property measurements of thin films using load deflection of composite rectangular membranes", Sensors and Actuators, 20, 135 (1989).

6. M.G. Allen, M. Mehregany, R.T. Howe, and S.D Senturia, "Microfabricated structures for the in situ measurement of residual stress, Young's modulus, and ultimate strain of thin films", Appl. Phys. Lett., 51,241 (1987).

\section{Present affiliation:}

*: Caliper Technologies Corp., 3180 Porter Dr., Palo Alto, CA 94304. **: Twente University, Enschede, The Netherlands.

***: Institute of Microtechnology, Univ. of Neuchatel, Switzerland. 